\title{
Akademievorträge \\ Wie einmal zusammenwuchs, was nicht zusammengehörte: Ein Blick auf die Entstehung der europäischen Universität
}

(Festvortrag in der öffentlichen Jahresfeier am 21. November 2009)

\section{Frank Rexroth}

Es ist zugleich eine Ehre und eine große Herausforderung, diesen heutigen Vortrag halten zu dürfen. Und ohne daß ich mir dies von Anfang an bewußt gemacht hätte, ist es vielleicht kein Zufall, daß auch ich mich ähnlich wie manche meiner Vorgänger aus den letzten Jahren entschlossen habe, gerade die Wissenschaft, genauer: die Universität zu meinem Gegenstand zu machen. Mögen die Akademien den Universitäten sowohl personell als auch institutionell aufs engste verbunden sein, so sind sie doch nicht deren Teil. Daher gibt mir dieser Vortrag die Gelegenheit, einen Schritt aus der Hochschule herauszutreten und die gebotene Minimaldistanz zu meinem Gegenstand einzunehmen, ohne daß ich damit zugleich meine kulturelle Rolle als Wissenschaftler aufgeben müßte. Ich hoffe, es führt nicht zu diplomatischen Komplikationen, daß ich von dieser Warte aus einen Blick auf die Universität werfe - was ich letztlich über sie sagen werde, ist jedenfalls nicht dazu angetan.

\section{Apologie und Klage, oder: Spreche ich von der Universität, dann spreche ich von mir selbst}

Freilich muß man sich vor bestimmten Gefahren hüten, wenn man die Hochschulen zu seinem Gegenstand macht. Denn man handelt dann mittelbar auch von sich selbst und seiner Lebenswelt, und so muß man sich vorsehen, daß nicht das Bedürfnis nach Apologie oder Klage die Feder führt. Das gilt auch für den Historiker und, was für viele vielleicht überraschend sein mag, gerade für den Historiker des europäischen Mittelalters. Denn die Mittelalterhistoriker sind die Experten für die Anfänge der europäischen Universität, für die ersten ca. 350 Jahre von deren Existenz, das heißt für den Zeitraum von deren Entstehung um das Jahr 1200 bis zu ihrer großen Vertrauenskrise, die mit der Reformation gekommen ist. Paß’ 
also auf, muß ich mir selber sagen, denn wer sich mit den Anfängen von Dingen beschäftigt, gerät unversehens in das Fahrwasser geistiger Traditionen, die gerade in den Anfängen der Dinge deren Kern erkennen wollen, alle ihre Stärken oder auch den bösen Wurm, der am eigenen Hochschullehrerdasein nagt. Apologie und Klage haben in der Tat den Blick auf die Anfänge der europäischen Universität gelenkt, den ich Ihnen angekündigt habe. Das ist aber leichter gesagt als getan. Denn die Frühgeschichte der Universitäten ist ein Thema von besonderem Reiz. Es markiert eine der sensibelsten Stellen in den historischen Selbstverortungen, innerhalb derer wir uns Rechenschaft über unsere politisch-soziale Identität ablegen. Die Universitäten entstanden an unterschiedlichen Orten in Europa, zunächst in Paris und Bologna und bald an weiteren wie Oxford und Montpellier, sozusagen aus wilder Wurzel. Sie wurden nicht gegründet, wie manchmal leichthin gesagt wird, sondern sie entstanden einfach - auf Neudeutsch: sie emergierten. Damit meint man, daß sie schließlich da waren, ohne daß man sie auf eine befriedigende Art kausal aus ihren Entstehungsbedingungen heraus erklären könnte. Der Zustrom von Schülern war an beiden frühsten Orten, in Paris wie in Bologna, groß, und wir können annehmen, daß eine derartige Konzentration junger Männer in einer Stadt zu sozialen Spannungen führte, auch zur zunächst unregulierten Konkurrenzsituation von Lehrern (magistri), die doch alle ihr Auskommen finden mußten. Sozialgeschichtlich gesehen ist es also wohl die Desorganisation von Versorgung und Regulierung in den Wirtsstädten und das Bedürfnis nach der Bildung eines Kartells, die dazu führten, daß sich am einen Ort - Paris - die Magister und ihre Scholaren, am anderen Ort - Bologna - nur die Scholaren zu Schwureinungen zusammentaten, zu universitates nach zeitgenössischem Verständnis. Universitas heißt zunächst ganz schlicht „Gruppe“ und wird schnell zum Rechtsterminus, der eine Korporation, einen verfaßten Personenverband, bezeichnet. Gemeint waren auch in diesem Fall von Anbeginn verfaßte Gruppen - sogar spezifisch verfaßte Gruppen, in die man eintrat, indem man einen promissorischen Eid leistete und schwor, die innere, selbst gegebene Ordnung zu befolgen und sich den selbst gewählten Organen der Universitas unterzuordnen.

"Selbst gegeben“, „selbst gewählt“, also Autonomie und Autokephaliedamit stehen die Universitäten keineswegs alleine da in der mittelalterlichen Welt, denn diese kennt entgegen heutigen Vorurteilen eine bunte, reichhaltige Kultur der selbstbestimmten Gruppenbildung sozusagen „von unten ", und man wird ihr nicht gerecht, wenn man mit ihr nur Kaiser und Päpste assoziiert. „Wir Wissenschaftler, wir Gelehrten“, so könnte man die Faszination gerade der institutionengeschichtlich arbeitenden Univer- 
sitätshistoriker auf den Punkt bringen, „wir Wissenschaftler haben es selbst geschafft, aus eigenem Antrieb und auf eigenes Risiko. Kein Fürst und kein Papst steht am Anfang unserer Universität, sondern wir haben uns unser Haus selbst erbaut". Spätestens seit dem Beginn der Moderne um 1800 war dieser Stolz Anlaß für einen heroisierenden, monumentalisierenden Blick auf die Universität und ihre Geschichte. Liberale Geister des 19. und des 20. Jahrhunderts schrieben diese Geschichte gerne, beispielsweise, wenn wieder einmal ein Jubiläum der eigenen Alma mater anstand. Weit über den Rahmen der Bildungsgeschichte hinaus reichte dieses heroische Narrativ, denn was lag näher, als es einzubetten in die großen Erzählungen vom Aufstieg Europas, von der Anbahnung der charakteristisch europäischen Vernunftdominanz in der Auseinandersetzung mit dem gelebten Glauben (wobei die Magister und Doktoren die Träger dieser Entwicklung waren), von der Entwicklung hin zu rational organisierten Staatswesen und ihrer Expertenstäbe (die ja zum großen Teil an den Universitäten ihr Fachwissen erlangten) und ganz generell von der Ausdifferenzierung eines sozialen Systems „Wissenschaft“, dem eigene Kommunikationsregeln und, damit verbunden, ein eigener Denkstil zugestanden werden. Alles hätte ganz anders kommen können in Europa, und wenn man sich fragt, in welchen Prozessen die Wissenschaft zu dem wurde, was sie im okzidentalen Europa geworden ist und in anderen Regionen der Welt nicht, dann wird man unweigerlich auf die Anfänge der Universität verwiesen, auf „Paris und Bologna“ als europäische Erinnerungsorte. Dies um so mehr, zumal wir ja wissen, als wie langlebig sich die Universität erwiesen hat. Woher rührt diese Langlebigkeit? Immerhin gab es seit den Anfängen im 13. Jahrhundert keine Phase der Universitätengeschichte, in der nicht mit beträchtlichem reformerischen Aufwand an den Hochschulen herumlaboriert worden wäre.

\section{Sir Richard will nicht mehr lehren}

Soviel zur Apologie der mittelalterlichen Universität, zu deren Monumentalisierung, von der ich eingangs sprach. Sie sehen, diese ist sozialgeschichtlich fundiert, insofern die Entstehung und Verstetigung sozialer Gruppen von Lehrenden und Lernenden an ihrem Ausgang steht. Wie steht es aber mit der Klage, das heißt mit der Rückführung gegenwärtiger Mißstände auf die Anfänge der Universitäten? Auch sie gibt es, wenngleich sie im Unterschied zu der monumentalischen Historie der Universitätengeschichte eher im Verborgenen wirkt, sozusagen subkutan. Diejenige Klage, die ich intellektuell für besonders spannend halte und mit der ich Sie daher in meinem nächsten 
Schritt bekannt machen will, ist nicht sozial-, sondern ideengeschichtlich fundiert. Sie betrifft die Inhalte der universitären Wissenschaft. Es sind einige der führenden Köpfe meiner Disziplin gewesen, die so dachten und schrieben - Peter Classen etwa oder auch Arno Borst und schließlich der 2001 verstorbene Sir Richard Southern, der mit dieser Sichtweise sein Spätwerk unter dem Titel „Scholastic Humanism and the Unification of Europe" grundierte. Ihn greife ich beispielhaft heraus. Ihm galt nicht die Frühgeschichte der Universitäten seit 1200, sondern das Jahrhundert davor, also das 12. Jahrhundert, als das "heroic age“ der mittelalterlichen Wissenschaft - so der Titel des 2001 erschienenen zweiten Bandes von „Scholastic Humanism and the Unification of Europe“. Southern spricht von einem recht homogenen wissenschaftlichen „Programm“, das die Gelehrten des 12. Jahrhunderts verfolgt hätten. Ihr Ziel sei ein „perfect system of knowledge" gewesen, das nach ihrem Dafürhalten vor dem biblischen Sündenfall schon einmal bestanden habe und nun wiedergewonnen werden müsse. Dieser beglückende Zustand habe bis etwa 1170 angehalten und sei dann für weitere 100 Jahre in eine Phase des routinierten Weiterforschens überführt worden. Diese Phase, also die Frühgeschichte der Universität, war für Southern viel weniger wertvoll als die Zeit davor, denn die Gelehrten des Jahrhunderts nach 1170 seien vornehmlich damit beschäftigt gewesen, Einzelheiten zu vertiefen oder Berge von Wissen zu akkumulieren. Pioniere waren sie keine mehr, das „scholastic programme“ wurde von ihnen verwaltet, aber nicht wirklich behauptet. Schließlich sei die Hoffnung der Gelehrten gänzlich geschwunden, daß man jenes „scholastic programme“ je erreichen könne. Die einen hätten sich frustriert in der akademischen Routine eingerichtet, die Scholastikkritiker hätten sich außerhalb der Hochschulen in mystischen und humanistischen Zirkeln zusammengetan. Was soll man davon halten? Unbestritten ist, daß das 12. Jahrhundert eine ungemein aufregende Zeit für bewegliche Geister gewesen ist, daß die jungen Männer, die sich zu den noch nicht universitär verfaßten Lehrer-Unternehmern in Paris und Bologna aufmachten, elektrisiert waren von der intellektuellen Aufbruchstimmung, die in diesen Städten herrschte. Tatsächlich stammen die meisten der großen, fundierenden Werke der scholastischen Wissenschaft, die bahnbrechenden Innovationen in den Bereichen der Philosophie, der Jurisprudenz, der Medizin sowie der Theologie, gerade aus diesem 12. Jahrhundert. Das heißt, daß sie der Entstehung der ersten Universitäten vorangehen, statt ihr Produkt zu sein. Dies gilt etwa für die neuerliche Erschließung des römischen Rechts, die von der Rezeption der Digesten seit dem letzten Viertel des 11. Jahrhunderts ausging; es gilt ebenso für Abaelards philosophisches Grundlagenwerk „Sic et non“ um 1122, für die Erschließung 
des Kirchenrechts durch das um 1140 verfaßte Decretum Gratiani und für die theologische Sentenzensammlung des Petrus Lombardus, wie sie in den 1150er Jahren in Paris heranwuchs; und es gilt letztlich für das Corpus medizinischer Einführungs- und Lehrschriften, das durch Übersetzungen aus dem Arabischen entstanden war. Wenn dies zuträfe, käme diese Aussage freilich einem vernichtenden Urteil gleich: die aufregendste und schöpferischste Phase in der Frühgeschichte der Universitäten wäre das Jahrhundert vor deren Entstehung gewesen. Man hat, wo man so dachte, von der Konstituierung der universitates zu Bologna, Paris, Oxford, Montpellier etc. um das Jahr 1200 so gesprochen, als stellten sie einerseits die Krönung einer wissenschaftlichen „Renaissance des 12. Jahrhunderts“ dar, als markierten sie aber zugleich deren Ende, als führten sie das Ereignis elektrisierender wissenschaftlicher Innovation in eine Struktur, in einen Zustand der Routine und der Epigonaliltät über. Die Pointe dieser Sicht ist (und dies sagten Southern und auch Peter Classen in aller Deutlichkeit), daß man für diesen Niedergang gerade die frisch entstandenen Universitäten mit ihrer institutionellen Struktur mit Fakultäten, Curricula, Prüfungsmodalitäten etc. verantwortlich machen könne, da diese beispielsweise der Naturforschung keinen adäquaten Raum geboten hätten. Wer Horoskope stellen, prophezeien, Arzneien herstellen oder angewandte Arithmetik betreiben wollte, hielt sich, so Southern, fortan lieber an die Höfe und die Klöster, „where individual scholars had freedom for observations and were not required to teach“.

\section{Die Klage über die Universität aus dem Geist der gestreßten Hochschullehrer}

Nochmals: „[...] where individual scholars had freedom for observations and were not required to teach ". - Dieser letzte Satzteil ist verräterisch, denn wir entdecken unsere eigenen Ressentiments darin: Der Wissenschaftsminister warnt: akademische Lehre, in hohen Quantitäten genossen, kann zum vorzeitigen Forschertod führen! Leicht erkennt man die Tragweite dieses Urteils, denn auch hier werden heutige Orientierungsbedüfnisse mit einem eigenen Geschichtsbild untermauert, wird über die Anfänge der Universität gesprochen und doch die zeitgenössische Krise der Universität gemeint. Denn zur Debatte gestellt wird damit die Universitätsförmigkeit der okzidentalen Wissenschaft schlechthin, also die Tatsache, daß die Wissenschaft bis heute überwiegend im Gehäuse von Universitäten und ihren Einrichungen angesiedelt ist, bewehrt durch Fakultäten, Curricula, Prüfungswesen und Promotionsrecht. Es geht mithin darum, daß 
die Dauerhaftigkeit der geistigen Prozesse, die in ihrer Gesamtheit Wissenschaft ausmachen, dadurch erzielt wird, daß man diese in komplexen Organisationen verankert hat. Ist dies für Forschung, die doch auf Innovation zielt und daher so beweglich wie möglich sein muß, tatsächlich von Vorteil? Wenn die Wissenschaft innovativ sein soll, so erwartet man, soll sie sich ganz an ihrem Gegenstand ausrichten können, soll sie keinen Ballast in Gestalt von Fächerstruktur, disziplinärer Selbstgenügsamkeit oder gar das ist der Gottseibeiuns - Verwaltung mitschleppen müssen. Als innovativen Forscher stellt man sich dann die originelle Einzelpersönlichkeit vor, einen „marginal man“ sozusagen, der möglichst von den Zwischenräumen zwischen ganz verschiedenen Wissensfeldern aus agiert und jede Spur von fachlicher Selbstgenügsamkeit vermeidet. Wir lieben diese Ikonen der Wissenschaft, stellen sie uns als unabhängige Geister in einer freimachenden Außenseiterposition vor und nicht als Dekane und Talarträger. Die Photographie, auf der Albert Einstein den Reportern die Zunge herausstreckt, fällt uns dazu ein, vielleicht gerade dann, wenn wir in der Strukturund Haushaltskommission unserer Fakultät sitzen und der Tagesordnungspunkt 13 „Anreizsysteme zum Energiesparen in den Räumen der Universität" aufgerufen wird. Energiesparen ist wichtig, himmelhergottnochmal, aber können sich damit nicht Andere befassen? Es gibt ja in der Tat zu denken, wie viele wissenschaftliche Innovationen auch in der Vormoderne schon von Menschen erbracht wurden, die der Universität nur sozusagen vorübergehend verbunden waren; auf Cusanus, Kopernikus und Kepler hat man in diesem Zusammenhang verwiesen, auf Galilei, Descartes und Spinoza, auf Pascal und Leibniz. Muß man, in Abwandlung des berühmten Wortes von Ernst Troeltsch, die Universitäten also als Schalen ansehen, die allmählich den Kern verholzen, den sie schützen? Bekäme der Wissenschaft eine lockere, vielleicht sogar ephemere Organisationsweise nicht besser? Es ist verführerisch, sich auszumalen, wie beweglich der Geist wäre, könnte er sich nur vom Körper befreien.

\section{Die Frage ist, was die Universität der Wissenschaft an-tut}

Die historische Forschung befindet sich, wenn sie an dieser Aufgabe gemessen wird, in einem Dilemma, denn die Sozialhistoriker schreiben im monumentalisierenden Stil eine Erfolgsgeschichte der Institution Universität, ohne sich viel um den Kernbereich der Wissenschaft zu kümmern (oder diesen doch vorschnell und unüberlegt mitzuheroisieren), und die Ideenhistoriker nehmen die mittelalterliche Geschichte als Kronzeugen dafür in 
Anspruch, daß sich der freie Geist von Institutionen nichts wirklich Gutes erhoffen darf. Handelt es sich dabei also um eine Sache der eigenen forscherlichen Präferenz „Sozial- oder Ideengeschichte“ oder gar um eine Sache des persönlichen Temperaments, ähnlich wie die mit dem Glas Wasser, das dem einen halb voll und dem anderen halb leer ist? Wenn ich die möglichen Gegenwartsbezüge dieser beiden Positionen hier andeute, dann keineswegs in der Absicht, sie bloßzustellen. Ihnen gerecht zu werden muß aber bedeuten, das hier mit nostalgischer Färbung angesprochene Problem für eine sozialhistorische Fragestellung operabel zu machen. Man wird es nicht gänzlich auflösen, aber ich will doch immerhin versuchen, einen Blick auf die Entstehung der disziplinär verfaßten Universität zu werfen und zugleich die Frage nach den wissenschaftlichen Inhalten ohne vorzeitige Heroisierung mit einzubeziehen. Das Problem läßt sich dann etwa folgendermaßen formulieren: Wenn sich der imposante, wieder und wieder erforschte Aufschwung der scholastischen Wissenschaft während des 12. Jahrhunderts im Milieu locker verfaßter, ephemerer „Schulen“ ereignet und wenn sich die Entstehung der okzidentalen Universitäten erst Jahrzehnte nach der Fertigstellung der großen, bahnbrechenden Arbeiten eines Peter Abaelard, eines Gratian und eines Petrus Lombardus ereignet - was bedeuten dann die Universität und ihre Einrichtungen für die Wissenschaft, was tun sie der Wissenschaft an, wie verändern sie sie?

Ich gehe dabei von dem Aspekt der Universitäten aus, den ich für den letztlich erklärungsbedürftigen halte, nämlich von der disziplinären Einteilung, der Clusterform der Universitäten, der Fakultätenstruktur, wobei die Fakultäten für spezifische Disziplinen oder Disziplinengruppen mit jeweils eigenem Textbezug, eigenen Regeln des Sagbaren und eigenen Denkstilen stehen. Diese Mehrzahl von Disziplinen war es, die im Gehäuse der Universität in Fakultäten festgeschrieben und mit Statuten, mit Studien- und Promotionsordnungen und der Unterscheidung der akademischen Grade verstetigt wurde. Dabei gab es abermals eine ganze Reihe von Strategien, das universitäre Ensemble der Wissenschaften zu strukturieren: hierarchisch, insofern beispielsweise zum Zweck der universitären Repräsentation die Rangfolge der Fakultäten festgelegt wurde; systematisch, insofern man das Voranschreiten von Fach zu Fach oder die Mindeststudiendauern für die einzelnen Fächer reglementierte; und disziplinär, insofern gruppenintern oder -extern (Päpste oder ihre Legaten, aber auch die Versammlungen der Professoren) die Grenzen des jeweils Sagbaren nach Fakultäten gesondert festgeschrieben wurden.

In meinem folgenden Schritt muß ich mich freilich auf Beispiele beschränken. Ich möchte einsetzen bei einer hochinteressanten zeitgenössi- 
schen Reflexion über die Relation der Wissenschaften zueinander, die aus der Mitte des 12. Jahrhunderts stammt, also aus der Phase unmittelbar vor der Entstehung der Universitäten. Die weiteren Schritte werden sich daraus ergeben.

\section{Vom Renommee der Lehrer zum Prestige der Schulen}

Zunächst also zur Situation um die Mitte des 12. Jahrhunderts, die geprägt ist durch zweierlei Aufschwung: den der Pariser Schulenlandschaft mit ihrem deutlichen Schwergewicht auf den Artes liberales und der Theologie und den der Bologneser Lehrer des Rechts. Beide Entwicklungen ereigneten sich gleichzeitig und waren in wenigen Jahrzehnten abgeschlossen. Als Peter Abaelard um 1133 nach Paris zurückkehrte, um sich dort zum zweiten Mal eine Schule aufzubauen, traf er eine veränderte Landschaft an: Während seines ersten Aufenthalts in den ersten Jahren des Jahrhunderts war der Ruhm der Schulen auf einen Einzelnen konzentriert gewesen, auf Wilhelm von Champeaux nämlich als den tonangebenden Lehrer, auf ein intellektuelles Alphatier, das herauszufordern den Ruhm des Nachwuchsgenies Abaelard begründet hatte. Nun aber, in den 1130ern, mußte Abaelard erfahren, daß man in der Stadt mittlerweile von einer Vielzahl von Lehrern sprach. Die Scholaren erörterten ihre Vorzüge und Schwächen, ihre Eitelkeiten, ihre Eifersucht, aber auch ihren Charme. Das Licht des Scholareninteresses ruhte jetzt auf vielen, und solange man die Freien Künste studierte, wollte man möglichst viele von ihnen gehört haben. Etwa zur selben Zeit, wohl um die Jahrhundertmitte, ging Vergleichbares auch in Bologna vor sich: Die Leitfigur der Vergangenheit war der herrisch auftretende, waffenführende iudex gewesen. Parteienbefragungen vor Gericht mit dem ganzen sozialen Gewicht seiner Persönlichkeit vornehmen zu können, dies hatte seine Qualität ausgemacht. War jemand ein wirklicher Kenner des Rechts, hatte man ihn durchaus als einen legis doctor bezeichnet. Dies hatte vor der Wende um die Jahrhundertmitte eben dies bedeutet: ein Kenner des Rechts zu sein, nicht unbedingt dessen Lehrer. Dies änderte sich nun, denn das neue Renomee der jetzigen legis doctores, der Berufsjuristen, gründete sich auf deren Publikations- und Lehrtätigkeit. Nicht mehr ihre ständische Autorität, nicht mehr, daß sie auf einem Pferd sitzend eine bella figura abgaben, sondern ihr Know-how sprachen für sie. Und begleitet wurde diese Veränderung von einer weiteren: der Ruhm der einzelnen Rechtsgelehrten strahlte auf Bologna selbst ab und führte dazu, daß sich schließlich der Ruf des Bologneser Rechtsstudiums insgesamt, in dieser Form eines Kollektivsingulars, verbreitete. Von „Paris und Bologna“ zu sprechen, wurde binnen 
kurzer Zeit geläufig. Hören wir eine dieser Stimmen zur Verschiedenheit und Geschiedenheit der Wissensbereiche. Es ist die des Johannes von Salisbury, der sich aus ca. zehn Jahren Distanz an seine Pariser Studienjahre von 1136 bis 1148 erinnert. Er betrat die Pariser Szenerie zu derselben Zeit, zu der sein Lehrer Abaelard die veränderte, weil mittlerweile aufgefächerte und vielgestaltig gewordene Pariser Schulenlandschaft antraf. Johannes hat diesen Bericht dem zweiten Buch seines 1159 beendeten „Metalogicon“ eingefügt. In gewisser Weise behandelt Johann diese Relation der Wissenschaften zueinander als den wichtigsten Aspekt seiner Laufbahn, denn sein Kapitel schildert im Ganzen eine Art biographisches Experiment: Es testet die Differenz zwischen dem Können und Wissen derer, die sich über zehn Jahre und mehr ausschließlich mit nur einer Wissenschaft beschäftigt haben (nämlich mit der neuen scholastischen Logik), und dem Autor selbst, der nach etwa drei Jahren logischer Schulung ein Unbehagen an der Selbstgenügsamkeit und Traditionsferne dieser Wissenschaft verspürte und sich anderen Wissensbereichen zuwandte: erst der Grammatik, den rechnenden Künsten des Quadrivium, der Rhetorik, dem Corpus der aristotelischen Schriften, schließlich der Theologie. Als er seine früheren Kommilitonen (veteres socios) wiedertraf, stellte er fest: Sie stellten immer noch dieselben Fragen wie vor Jahr und Tag und gaben immer noch dieselben Antworten! Sein Urteil hätte er kaum schärfer treffen können: Inuenti sunt qui fuerant et $u b i$, ich traf sie als dieselben und am selben Ort an, an dem ich sie verlassen hatte, im buchstäblichen und im übertragenen Sinne reglos. Der Wechsel von Disziplin zu Disziplin wird hier als notwendige Bedingung dafür eingebracht, wissenschaftliche Urteilskraft überhaupt zu entwickeln. Wer nur die Logik versteht, versteht auch diese nicht. Transdisziplinarität, so könnte man leicht anachronistisch sagen, ist hier das Gebot der Stunde, denn andernfalls wäre der geistige Impuls, der von der neuen scholastischen Logik ausging, zu einer sterilen Beschäftigung in einem engen Kreis von Kennern und Liebhabern geworden.

\section{Wieso Juristen und Philosophen einander nicht schätzen}

Dies ist aber nicht der einzige Fächerwechsel, der in dem Kapitel beschrieben wird, und der zweite, der weniger organisch verläuft, ist für meine Belange der wichtigere, denn er weist auf die Universität der Zukunft voraus. Johann berichtet von ihm, als er von der Verwandlung seines Dialektiklehrers Alberich von Paris spricht, der zunächst ein Ausbund an dialektischer Selbstgenügsamkeit war. Nach Jahren der Lehrtätigkeit an der Seine 
ging Alberich nach Bologna und wandte sich dort dem gelehrten Recht zu. Dabei machte er eine überraschende Erfahrung: denn es kam für ihn im italienischen Rechtsunterricht darauf an, zunächst einmal zu verlernen, was er zuvor bei den Pariser Artisten nicht nur gelernt, sondern sogar gelehrt hatte. Dadurch habe er sich gänzlich verändert: Nam postea unus eorum [Albericus, F.R.] profectus Bononiam dedidicit quod docuerat. Siquidem et reuersus dedocuit. Die Erfahrung des anderen Fachs krempelte ihn so sehr um, daß man ihn nach seiner Rückkehr nach Paris als Lehrer kaum wiedererkannte. Der Übertritt von einer Disziplin zur anderen - hier: von den Artes zu den Jura - scheint also weniger reibungslos verlaufen zu sein als der Fächerwechsel innerhalb der Artes liberales oder der von der Logik zur Theologie. Hochinteressant an Johanns Charakterisierung dieser Situation (,er verlernte, was er zuvor gelehrt hatte") ist, daß er sich derselben Diktion vom notwendigen Vergessen des artistischen Wissensballasts bedient, den die gelehrten Juristen an den zeitgenössischen Bologneser Schulen, die Glossatoren also, selbst bemühen: Placentinus etwa, der im Exordium zu seiner um 1165 (also ca. sechs Jahre nach Johanns Text) verfaßten Institutionensumme betont, daß ein Jurist Grammatik und Logik nicht lernen, sondern besser ver-lernen solle, und Jahrzehnte später Azo, der dieses Urteil in der Einleitung seiner eigenen Institutionensumme wiederholt. Wie ist diese überraschende Forderung einzuordnen? Die Gleichsetzung von scholastischer Dialektik mit Sophisterei und leerem Geschwätz war unter den (juristischen) Glossatoren ein gängiges Klischee, so in der Glosse „Grammatici“ zu Inst. 1,25,15, bei Odofredus, Pillius und eben auch Placentinus. Bei den „Artes“ und zumal der Logik, in der es um solche Themen wie die Natur der Welt und die Rundung des Firmaments gehe, handele es sich um Pseudophilosophie, so der Placentinusschüler Otto Papiensis. Bei Azo mag ein solches Urteil nicht weiter verwundern, galt er doch als artistischer Ignorant: Sed de domino Az [one] non miror, so Odofredus, quia non fuit extremus in artibus, licet in scientia nostra fuerit summus [...]. Doch derlei von Placentinus zu hören, der offenbar literarisch ausgesprochen interessiert und auch bewandert war, ist durchaus bemerkenswert, immerhin ist dieser der Autor eines literarisch ambitionierten „Sermo de legibus“. Hermann Kantorowicz hat ihn daher als einen „classical scholar as well as a jurist" bezeichnet. Wer das Recht studieren will, sollte also besser sein Artistenwissen an der Garderobe abgeben! Man ist versucht, sich auszumalen, wie Johann von Salisbury an dieser Stelle seines „Metalogicon“ auf ein Urteil zurückgreift, das der Gegenstand seines knappen Berichts, sein ehemaliger Lehrer Alberich, in Bologna aufgeschnappt, mit an die Seine zurückgebracht und dort kolportiert hat. Und man ist ferner versucht, sich vorzustellen, wie dasselbe 
Bologneser Bonmot der 1130er Jahre auf anderen Wegen in das Oeuvre der Glossatoren Placentinus und Azo gelangte. Auf der Seite der Juristen hat man sie dann einmal als Ausdruck von Herablassung gegenüber den Philosophen zu lesen - immerhin litten gerade die beiden Bologneser Großprofessoren Placentinus und Azo nicht gerade unter drückenden Minderwertigkeitskomplexen. Dem Juristenstolz verwandt ist der Dünkel derer, die sich in der Verwaltung nützlich machen wollen. Richard FitzNigel schreibt zu Beginn seines „Dialogus de Scaccario“: „Wer an Erfindungen Freude hat, wer die Flucht in Subtilitäten sucht, der möge sich an Aristoteles und die platonischen Schriften halten. Schreib Du nichts Subtiles, sondern Nützliches“. Angemessener scheint mir aber, die Rede vom Vergessenmüssen des philosophischen Denkens anders zu lesen: Die Beschäftigung mit der Grammatik und der Dialektik kann durchaus sinnvoll sein, doch den Artisten, die die Bologneser Rechtsschulen betreten, muß es um die Aneignung eines gänzlich anderen, nämlich juristischen Denkstils gehen. Vor dem Hintergrund der heutigen wissenschaftshistorischen Meistererzählungen ist diese Lesart überraschend, da diese Meistererzählungen die großen geistigen Impulse des 12. Jahrhunderts als einen durch und durch homogenen Vorgang präsentieren, nach dem Abaelards „Sic et non“ und Gratians "Concordantia“ oder die Rezeption der Digesten und der theologische Sentenzenkommentar des Petrus Lombardus leicht in einem Atemzug genannt werden. Eben die Glattheit dieser Meistererzählung von der „Renaissance der Wissenschaften im 12. Jahrhundert" verführt dazu, anzunehmen, daß es sich bei diesem Vorgang um ein einheitliches, homogenes und alle Wissenschaften in einen Verbund bringendes „revival“ gehandelt habe, aus dem die Universität dann irgendwie zwingend hervorgegangen sei. Die Zeitgenossen sahen dies offenbar anders, wie ein Blick auf die Juristen belegt. Sowohl die Gelehrten des römischen als auch die des Kirchenrechts waren während des 12. Jahrhunderts im Gegenteil bestrebt, ihre jungen Disziplinen gänzlich autochthon erscheinen zu lassen, das heißt zu zeigen, daß das Recht, und nur das Recht, die Rechtswissenschaft hervorgebracht habe: magis [...] credere debemus Romanae legis auctoritati quam rhetoricae, so hat man gesagt, man muß der Autorität des römischen Rechts mehr Glauben schenken als der Rhetorik. Dies war eine disziplinenpolitisch essentielle Abgrenzung, denn in Wirklichkeit war es ganz anders gewesen: Die rudimentäre Kenntnis des römischen Rechts in der Zeit vor der Wiederentdeckung der Digesten war in Wirklichkeit vor allem über rhetorischen Unterricht vermittelt worden. Die frühe Legistik redet hier einen Kontinuitätsbruch herbei, der sie von den Artes liberales vollständig emanzipieren soll, und in ihren frühen Selbstbeschreibungen bringt sie diese Botschaft an ihre Scholaren: „Wo- 
her kommst du? Aus Paris? Dann gebe ich Dir den Rat: Vergiß am besten alles, was du dort gelernt hast“. Die Glossatoren der ersten Stunde wollten nicht die Verwandten von Berufsrednern sein. Ähnliches gilt auch für die Kanonistik: Gratian selbst warnt die Interpreten heiliger Schriften vor den Fallstricken der Dialektiker. Mit Sophismen und dialektischer Kunst bedrückten Häretiker die Männer der Kirche, so der Autor der „Concordantia“. Es ist sicher kein Zufall, daß man erst später diese selbstgewählte Selbstreferentialität durchbricht und die zeitgenössische Philosophie ernster zu nehmen beginnt. Wir befinden uns dann im Zeitalter der Universitäten, und die Juristen sehen sich vor neue Aufgaben gestellt, denn mit der weiterschreitenden Verwissenschaftlichung des Rechts beginnen sie, sich über die vorjuristischen Grundlagen juristischer Praxis, also etwa über die Rechtsdogmen, Gedanken zu machen. In diesem Stadium erst erkennen sie den Nutzen der philosophischen Reflexion, so beispielsweise über die principia. Dies ist bei Azo (gest. wohl nach 1229) der Fall, und diesem schließt sich Baldus de Ubaldis (gest. 1400) an: Wer wissen wolle, was aus den Prinzipien hervorgegangen sei, müsse die Prinzipien selbst kennen; es seien nämlich die ersten Prinzipien wie Pforten, an denen man sich nicht irren dürfe, denn kleine Irrtümer bei den Prinzipien wüchsen sich zu großen Irrtümern am Schluß aus. Hier haben Aristoteles und Thomas von Aquin Pate gestanden, hier befinden wir uns auch tief in der Zeit der Universitäten, also in der Ära der institutionell bewehrten Interdisziplinarität.

\section{Wahrheit und Nützlichkeit}

Würde man die Blickrichtung umkehren - was ich aus Zeitgründen nicht tun kann - und nicht mehr durch die Brille der Juristen auf die Artisten/ Philosophen, sondern von den letzteren aus auf die Juristen schauen, dann begegneten wir selbst bei den aufgeschlossensten Geistern wie dem Spanier Dominicus Gundisalvi (gest. 1190 oder später) gleichfalls klischeehaften Vorstellungen von zwei Kulturen der Gelehrsamkeit. Sich selbst schreiben die Philosophen dann zu, die vernunftgemäße Erkenntnis der Wahrheit zum Ziel zu haben (mit den Wegstationen der Physik, der Mathematik und der Metaphysik), den anderen aber (nämlich den Rhetoren, Poeten und Juristen) das Streben nach sozialer Nützlichkeit. Dabei kommen die Nützlichkeitswissenschaften häufig nicht gut davon - ein ganzes Arsenal von Unterstellungen prägt die Sichtweisen schon im 12. Jahrhundert: Die Klagen über den Karrierismus gerade der Juristen, ihre Habgier und ihre Versessenheit darauf, die Nähe zur Macht zu suchen. Genau diese bei- 
den Diskurse, der um die Wahrheit und der um die Nützlichkeit, wurden mit der Entstehung der Universitäten dauerhaft aneinandergeschweißt und einander ausgesetzt. Lassen Sie uns einen Augenblick zurückblicken. Es scheint nach dem Gesagten aus der Eigenlogik des hochmittelalterlichen Schulenbetriebs heraus durchaus folgerichtig zu sein, daß an den Universitäten Frankreichs, zumal in Paris, die Artes und die Theologie dauerhaft miteinander verbunden wurden. Es scheint ebenso folgerichtig zu sein, daß die italienischen Scholarenuniversitäten der Frühzeit so stark juristisch dominiert waren; erst allmählich trifft man dort Artisten- und Theologenfakultäten (oder genauer: Artisten- und Theologenuniversitäten) an - sie wirken wie ein dazugezimmerter Anbau an einem bereits Bestehenden. Aber man muß es als ein rundherum kontingentes Ereignis von im nachhinein betrachtet globaler Bedeutung ansehen, daß aus diesen beiden, aus lokaler Eigentradition heraus zu erklärenden Entwicklungen mit den Universitäten ein Ganzes wurde. Spätestens zu dem Zeitpunkt, zu dem in Europa Universitäten in Kenntnis von deren Möglichkeiten geplant und gegründet wurden (also seit ca. 1230), begann das idealtypische Vier-FakultätenModell zur Norm zu werden: Artisten, Mediziner, Juristen, Theologen. Damit aber wurden die höchst unterschiedlichen Wissenschaftskonzeptionen der Artisten, der Theologen und wohl auch der Mediziner auf der einen und der Juristen auf der anderen Seite dauerhaft aneinandergebunden. Dies ist nämlich meine These: daß das prägende Element der okzidentalen Universität in der institutionellen Verschränkung dieser eigentlich auseinanderstrebenden Impulse liegt und daß erst diese institutionelle Verschränkung (das heißt vornehmlich: die Fakultätenstruktur der Universität) die Ehe der Wahrheitswissenschaften mit den Nutzanwendungswissenschaften herbeiführte, das Doppel von Orientierungs- und Verfügungswissen, das spannungsreiche, aber nie mehr aufgehobene Miteinander eines philosophischtheologischen und eines im weitesten Sinne politischen Diskurses. Die Pointe dieser Sichtweise ist, daß an den Universitäten seit dem 13. Jahrhundert zusammenwächst, was der Eigenlogik seiner Bestandteile nach keineswegs zusammengehört hat. War diese Grundspannung aber erst einmal etabliert, dann hielten die Universitäten auch weitere, gleichfalls divergierende Wissenschaftskonzepte aus, etwa die Spannung zwischen den an ratio und auctoritas orientierten Wissenschaften und solchen, in denen das Experiment als der Ort kontrollierter, wiederholbarer Erfahrung das Feld beherrschte. Die Grundspannung des Ursprungskonflikts begegnet uns später in neuen Konfigurationen wieder, in konkurrierenden Wahrheitskonzeptionen etwa, so zum Beispiel dort, wo man die Vielfalt der Phänomene einerseits auf Grundprinzipien und Gesetze zurückführen, andererseits aber 
das Individuelle in seiner Einzigartigkeit erfassen will: „Philosophie“ versus „Geschichte"(etwa: Naturgeschichte) nennt man das im 18. Jahrhundert, in der Moderne meint man dasselbe und spricht von den Natur- und den Geisteswissenschaften, von nomothetischen oder ideographischen Wissenschaften, vom Erklären und vom Verstehen. So gesehen, übt die Institutionalität der Universität, genauer: deren Clusterstruktur, einen heilsamen Druck auf die Struktur der Wissenschaften mit ihrer Tendenz zur immer weiter voranschreitenden Ausdifferenzierung und Selbstreferentialität aus. Was sie den Fächern an-tut, ist meines Erachtens unter diesem Vorverständnis im jeweiligen Einzelfall zu prüfen. Jedenfalls hat man im Gefüge der Universitäten schon seit dem 13. Jahrhundert auf Seiten der reflexiv orientierten, um einen Wahrheitsbegriff herum organisierten Wissenschaften stets mit den Ingenieuren sozialer Wirklichkeit in der Nachbarschaft zu rechnen, die auf Nutzanwendung, „Verfahren“ und Lebensweltlichkeit bedacht waren, und umgekehrt. Die Spezifik des sozialen Systems „Wissenschaft“ in den lateineuropäischen Gesellschaften schuldet dieser inhärenten Dissonanz wahrscheinlich ihr entscheidendes Moment. Und auch die Rolle des Funktionssystems „Wissenschaft“ in unseren Gesellschaften ist durch seine Universitätsförmigkeit erst ermöglicht worden. Damit meine ich die Ambivalenz der Wissenschaft dem Gesamtsystem „Gesellschaft" gegenüber, die im Vergleich mit anderen Funktionssystemen wie der „Politik“ etwa sichtbar wird: Auf der einen Seite verstehen wir die Wissenschaft nämlich nicht ich zitiere hier Niklas Luhmann - als ,freischwebende[n] Weltbeobachter“, „sondern als wissensförderndes Unternehmen der Gesellschaft und genauer: als Funktionssystem der Gesellschaft“; zugleich aber stellen wir „eine traditionsbestimmte Vorrangbehauptung“ auf - und dies „,nicht, wie im Falle der Politik, für eine Position in der Gesellschaft, sondern für eine Position über der Gesellschaft". Diese Ambivalenz: die erste Beobachterin der Gesellschaft und zugleich in der Gesellschaft zu Hause zu sein, konnte nur die janusköpfige Universität aushalten, das Haus der Philosophen und der sozialen Ingenieure. Keine Spezialschule, keine Grande école wäre hierzu dauerhaft geeignet gewesen. 